\title{
Pediatric myocardial protection: Where do we stand?
}

Bradley S. Allen, MD

See related article on page 67
From the Department of Cardiothoracic and Vascular Surgery, The University of Texas at Houston, Memorial Hermann Children's Hospital, Houston, Tex.

Received for publication March 9, 2004; accepted for publication March 22, 2004.

Address for reprints: Bradley S. Allen, MD, Professor of Cardiothoracic and Vascular Surgery, Chief, Pediatric Cardiac Surgery, The University of Texas at Houston, 6431 Fannin St, MSB 1.214, Houston, TX 77030 (E-mail: Bradley.Allen@uth.tmc.edu).

J Thorac Cardiovasc Surg 2004;128:11-3

$0022-5223 / \$ 30.00$

Copyright $\odot 2004$ by The American Association for Thoracic Surgery

doi:10.1016/j.jtcvs.2004.03.017

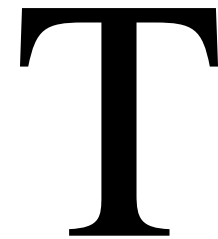

he objectives of every cardiac operation, whether adult or pediatric, are technical success and absence of iatrogenic injury from inadequate myocardial protection. To obtain the best results, the surgeon should select a method of protection only after learning how the cardioplegic method was developed, as well as how to benefit from its advantages, and how to avoid inappropriate use by recognizing the disadvantages. The choice for protection is similar to the selection of a structural technique for surgical repair of an underlying cardiac lesion. Failure of either modality is unacceptable, because both are equally important if the patient is to receive the full benefit from the surgical correction. Improved myocardial protection is not a phase of surgical development; it is intrinsic to the repair of congenital cardiac defects.

Despite major advances in the technical aspects of surgical repair, pediatric myocardial protective techniques remain relatively unchanged. Moreover, there are currently few articles in the major journals on pediatric myocardial protection. This would imply that current techniques provide optimal and complete preservation of the pediatric heart and that there is little need for further research to improve protection methods. However, pediatric patients continue to need mechanical assist devices, as well as prolonged inotropic support or an open chest despite a "technically perfect repair." Moreover, perioperative myocardial damage with low cardiac output remains the most common cause of morbidity and death after repair of congenital lesions. ${ }^{1-3}$ Cardiac damage from inadequate myocardial protection can prolong hospital stay and result in delayed myocardial fibrosis, leading to cardiac dysfunction months to years later. ${ }^{1}$ Protection of the immature heart is further complicated by a reduced response to inotropic agents relative to the adult heart. ${ }^{2-4}$ Thus preservation of myocardial function in immature hearts assumes even greater importance, because a perioperative insult is both less well tolerated and more difficult to treat.

In general, most changes in pediatric myocardial protection have been derived from the adult cardiac experience. However, in view of the structural, functional, and metabolic differences, extrapolation of adult cardioprotective strategies to the pediatric patients is fundamentally imprudent and potentially harmful. ${ }^{2-7}$ Myocardial protective strategies and cardioplegia solutions must be studied in the immature heart if pediatric protection is to be truly optimized.

In this issue, Modi and associates ${ }^{8}$ report a randomized clinical trial comparing crystalloid with blood cardioplegia in pediatric patients. Demonstrating differences between cardioplegic techniques is often difficult in the clinical setting because, unlike in the experimental laboratory where variables can be precisely controlled and measured, patients are not uniform, and the measurements of damage are less precise. In the experimental laboratory all hearts are subjected to the same preintervention stress, they undergo identical periods of ischemia, their anatomy is not altered, and postoperative measurements are precise and sensitive to detect damage. This makes it easy to demonstrate differences in protection strategies. In contrast, hearts in clinical studies are all different before surgery, they are anatomically altered (repaired), during surgery their crossclamp times are varied, and the postoperative measurements are far less precise. This makes it much more difficult to demonstrate clinical differences unless they are profound, even though significant myocardial damage may have occurred. 
Another problem in assessing clinical adequacy of myocardial protection is when to examine patient outcome and cardiac function, because direct assessment of myocardial damage is much more difficult than in the experimental laboratory. Ideally we should examine cardiac function not only in the perioperative period but several years later. A heart with nearly normal preoperative function may come off bypass, and the patient may go home despite rather extensive myocardial damage, giving the surgeon the false impression the protection was excellent. Nonetheless, perioperative myocardial damage may affect the long-term outcome and lead to late cardiac dysfunction months to years after surgical repair. ${ }^{1}$ This may be particularly important in patients who require several surgeries to correct their defects and may explain, for example, the late cardiac dysfunction in patients with hypoplastic left heart syndrome following their Fontan procedure.

Despite these limitations, the data presented by Modi and associates ${ }^{8}$ support several experimental and clinical studies and demonstrate that blood cardioplegia with a warm reperfusate provides better protection than crystalloid or cold blood cardioplegia alone. ${ }^{7,9,10}$ However, this improvement in protection was only seen in cyanotic patients. This is not particularly surprising, because hypoxic hearts are less tolerant of surgical ischemia and more dependent on the method of protection. ${ }^{2,5,7,9}$ Nevertheless, it is surprising that the study only examined relatively older, low-risk pediatric patients with essentially normal cardiac function. They excluded neonates and patients with hemodynamic instability, cases in which clinical differences in protection strategies are more likely to be identified. These are also the patients most dependent on optimal protection, as well as the group most likely to exhibit differences in protection strategies relative to adult (mature) hearts. Therefore they are probably the most important patients to include in studies of pediatric cardioprotection techniques.

In any investigation of myocardial protection, it is critical that the composition of the cardioplegia solution and the method of delivery be closely examined in interpreting the results. In their study, Modi and associates ${ }^{8}$ used St Thomas' Hospital solution (Plegisol) cardioplegia, either alone or mixed with blood, to compare crystalloid to blood cardioplegia with or without a warm reperfusate. However, these cardioplegic formulations may not provide an optimal test of blood versus crystalloid cardioplegia. Plegisol was specifically developed to work best as a crystalloid solution, with the constituents adjusted to provide optimal protection at these levels. ${ }^{2,11}$ Likewise, blood cardioplegic solutions were formulated to provide the ideal levels of the various components only after they are mixed with blood. ${ }^{1}$ In other words, blood cardioplegia is not just any crystalloid solution added to blood. Mixing Plegisol with blood alters the concentrations of its components and as such may not provide the same level of protection as when Plegisol is used as a crystalloid solution, or as cardioplegia solutions designed to be mixed with blood. Could the lack of difference seen by Modi and associates ${ }^{8}$ between blood and crystalloid cardioplegia therefore be the result of not using a blood cardioplegia solution explicitly developed for that purpose?

Perhaps of more concern is the composition and delivery of the warm reperfusate used in their study. Just as blood cardioplegia is not just any cardioplegic solution added to blood, a warm reperfusate is not just any solution given warm. Warm reperfusate solutions are specifically formulated to limit the reperfusion injury, and their composition is vastly different from the cardioplegic solution used for maintaining myocardial arrest. ${ }^{1,7,10}$ Reperfusate solutions are also usually given for at least 4 to 5 minutes, because it takes time to reverse any ischemic damage that may have occurred during the period the aorta is clamped. ${ }^{1,7,10} \mathrm{In}$ contrast, Modi and associates ${ }^{8}$ delivered their warm reperfusate solution for only 2 minutes, and they did not use a reperfusate solution specifically formulated for this purpose. This almost certainly limited the effectiveness of this modality and may be why an even greater benefit was not observed.

Substrate enhancement of the reperfusate solution is another factor that must be considered. My own group previously showed that infusing a warm non-substrate-enriched blood cardioplegic reperfusate for 4 to 5 minutes partially improved postbypass function and metabolic recovery in hypoxic piglets undergoing 70 minutes of arrest. ${ }^{10}$ This parallels the findings by Modi and associates, ${ }^{8}$ who did not use substrate enhancement. However, we subsequently found that enriching the warm cardioplegic reperfusate with the amino acids aspartate and glutamate vastly improved its efficacy, resulting in complete myocardial and metabolic recovery. ${ }^{7,10}$ The increased sensitivity to surgical ischemia we saw in our hypoxic neonatal piglets also parallels the findings in cyanotic infants and chronically hypoxic animals. ${ }^{2,5,12-14}$ Moreover, Taggart and associates ${ }^{15}$ recently demonstrated that both acyanotic and cyanotic infants undergoing corrective surgery are more prone to a reperfusion injury than are adults. This may explain why Chaturvedi and coworkers, ${ }^{16}$ who used a conductance catheter to measure pressure-volume loops, demonstrated postoperative ventricular dysfunction even in infants undergoing simple atrial septal defect repair when the heart was protected by cold cardioplegia alone. Use of this sensitive modality revealed that myocardial damage may occur even during repair of the simplest pediatric lesions but it may not be detected by the usual clinical parameters, such as length of stay or days in the intensive care unit. Substrate enrichment of the warm cardioplegic reperfusate may therefore have improved the results seen by Modi and associates ${ }^{8}$ in both acyanotic and cyanotic patients. 
Critical analysis of the adenosine triphosphate (ATP) data presented by Modi and associates ${ }^{8}$ raises another concern. Even though most patients were at low risk, postarrest ATP levels were reduced in every group. This implies that all three cardioplegic methods provided inadequate protection. A fall in postreperfusion ATP levels even in low-risk acyanotic patients with simple lesions demonstrates the vulnerability of the pediatric heart to ischemia and the need for improved methods of protection for all pediatric patients. It implies that although the patients may do fine clinically, myocardial damage is present. Perhaps if a blood cardioplegia and warm reperfusate solution specifically developed for this purpose had been given for an appropriate time interval, both acyanotic and cyanotic patients would have had complete return of ATP levels, as occurred even in severely stressed hypoxic neonatal piglets. ${ }^{7,10}$

In conclusion, the study by Modi and associates ${ }^{8}$ is important, because it is one of only a small number of studies to investigate myocardial protection strategies in pediatric patients. Only from studies such as this are we able to determine the optimal method of protecting the pediatric heart, instead of blindly, and often uncritically, adopting untested adult cardioplegic strategies. However, we must look with caution to the composition of the cardioplegia solution and the method of delivery in interpreting the results. It is important that cardioplegic solutions designed and tested for a specific purpose be used in any study of cardioplegic protection. These solutions should be delivered according to established experimental protocols and, most importantly, the aim must be complete preservation of metabolic and functional parameters. A fall in postarrest ATP or other metabolic levels should be looked at as a protection failure, in the same way that a residual ventricular septal defect or atrioventricular valve regurgitation is a technical failure. Optimal protection is as important as the technical aspects of the repair, and until we have developed cardioplegic techniques that completely avoid myocardial damage, we must continue to actively investigate new cardioplegia strategies. Ideally, this important study will spur others to examine protection strategies in both high- and low-risk pediatric populations and will reaffirm the importance of protection in determining the success of a pediatric operation.

\section{References}

1. Buckberg GD, Allen BS. Myocardial protection management during adult cardiac operations. In: Baue AE, Geha AS, Hammond GL, Laks H, Naunheim KS, editors. Glenn's thoracic and cardiovascular surgery. 6th ed. New York: Appleton \& Lange; 1995. p. 1653-87.

2. Castaneda AR, Jonas RA, Mayer JE, Hanley FL. Myocardial preservation in the immature heart. In: Castaneda AR, Jonas RA, Mayer JE, Hanley FL, editors. Cardiac surgery of the neonate and infant. Philadelphia: WB Saunders; 1994. p. 41-54.

3. Hammon JW. Myocardial protection in the immature heart. Ann Thorac Surg. 1995;60:839-42.

4. Romero TE, Friedman WF. Limited left ventricular response to volume overload in the neonatal period. Pediatr Res. 1979;13:910-5.

5. Silverman N, Kohler J, Levitsky S, Pavel D, Fang R, Freinberg H. Chronic hypoxemia depresses global ventricular function and predisposes to depletion of high energy phosphates during cardioplegic arrest. Ann Thorac Surg. 1984;37:304-8.

6. Friedman WF. The intrinsic physiologic properties of the developing heart. Prog Cardiovasc Dis. 1972;15:87-111.

7. Allen BS, Barth MJ, Ilbawi MN. Pediatric myocardial protection: an overview. Semin Thorac Cardiovasc Surg. 2001;13:56-72.

8. Modi P, Suleiman MS, Reeves B, Pawade A, Parry AJ, Angelini GD, et al. Myocardial metabolic changes during pediatric cardiac surgery: a randomized study of 3 cardioplegic techniques. J Thorac Cardiovasc Surg. 2004;128:67-75.

9. Bolling KS, Allen BS, Wang T, Ramon S, Feinberg H. myocardial protection in normal and hypoxically stressed neonatal hearts: the superiority of blood versus crystalloid cardioplegia. J Thorac Cardiovasc Surg. 1997;113:994-1005.

10. Kronon M, Allen BS, Raham SK, Wang T, Rayyab NA, Bolling KS. Reducing postischemic reperfusion damage in neonates using a terminal warm substrate enriched blood cardioplegic reperfusate. Ann Thorac Surg. 2000;70:765-70.

11. Hearse DJ, Stewart DA, Braimbridge MV. Myocardial protection during ischemic cardiac arrest. The importance of magnesium in cardioplegic infusates. J Thorac Cardiovasc Surg. 1978;75:877-85.

12. Fujiwara T, Kurtts T, Anderson W, Heinle J, Mayer JE. Myocardial protection in cyanotic neonatal lambs. $J$ Thorac Cardiovasc Surg. 1988;96:700-10.

13. Del Nido PJ, Mickle DA, Wilson GJ, Benson LN, Weisel RD, Coles JG. Inadequate myocardial protection with cold cardioplegic arrest during repair of tetralogy of Fallot. J Thorac Cardiovasc Surg. 1988; 95:223-9.

14. Teoh KH, Mickle DA, Weisel RD, Li R, Tumiati L, Coles JG. Effect of oxygen tension and cardiovascular operations on the myocardial antioxidant enzyme activities in patients with tetralogy of Fallot and aorta-coronary bypass. J Thorac Cardiovasc Surg. 1992;104:159-64.

15. Taggart D, Hadjinikolas L, Hooper J, Albert J, Kemp M, Hue D. Effects of age and ischemic times on biochemical evidence of myocardial injury after pediatric cardiac operations. J Thorac Cardiovasc Surg. 1997;113:728-35.

16. Chaturvedi R, Lincoln C, Gothard J, Scallan M, White P, Redington A. Left ventricular dysfunction after open repair of simple congenital heart defects in infants and children: quantitation with the use of a conductance catheter immediately after bypass. J Thorac Cardiovasc Surg. 1998;115:77-83. 